\title{
Quantitative Study of an Incommensurate Grain Boundary Using Aberration Corrected Microscopy
}

\author{
A. Gautam*, T. Radetic*, F. Lancon** and U. Dahmen* \\ *National Center for Electron Microscopy, Materials Sciences Division, LBNL, Berkeley, CA \\ 94720 \\ **Laboratoire de Simulation Atomistique (L_Sim), SP2M, INAC, CEA, 38054 Grenoble, France
}

Understanding grain boundary (GB) behavior is important due to their strong influence on the properties of a polycrystalline material. High resolution electron microscopy (HREM) is an invaluable tool to study GB structures because it allows a detailed analysis of their local structure at the atomic level. Recent advances in aberration corrected transmission electron microscopes have made it possible to image these GBs with sub-Angstrom resolution and to determine the positions of atomic column with sufficient accuracy to compare experimental data with predictions from atomistic simulations.

In the present work, an incommensurate $90^{\circ}<110>$ tilt GB in $\mathrm{Au}$ was studied using aberration corrected HREM. The samples were prepared by physical vapor deposition of high purity Au onto $<100>$ Ge substrates. The Ge substrate was later dissolved in a 1:1 solution of hydrofluoric acid and hydrogen peroxide, resulting in free standing Au films [1]. This method produced $<110>$ oriented bicrystals with a crystal misorientation of $\sim 90^{\circ}$. Selected GBs were imaged using the TEAM microscopes at the National Center for Electron Microscopy. The images were processed using the MacTempas software to fit a Gaussian peak to each atomic column followed by lattice fitting. Atomic columns were identified by a template matching procedure, using an average motif from the image itself as a template. Two separate lattices on either side of GB were defined through a least squares fit in an undistorted region at a distance from the GB. Finally, the displacement of atomic columns or peaks from this lattice was calculated. As an example, Figure 1a shows an HREM image of an incommensurate $90^{\circ}<110>$ tilt grain boundary in gold and Figure $1 \mathrm{~b}$ shows a magnified view of the displacement vectors calculated using this procedure.

Since high resolution images limit the length of GBs that can be observed within a single micrograph, it is essential to combine the data obtained from various high resolution images in order to obtain sufficient data for meaningful statistics. This becomes an issue especially in case of incommensurate GBs due to the lack of periodicity, which otherwise can be used to combine data from different micrographs. One possible way to overcome this limitation is by using Aubry hull functions [2,3], which involves measuring atomic displacements relative to an unrelaxed periodic reference lattice. These displacements are then plotted modulo the periodicity along the grain boundary in the opposite grain, i.e. atomic positions that are farther than the periodicity in the opposite grain are folded over by subtracting integer multiple of periodicity in the opposite grain. This procedure was followed to plot the data obtained from HREM images of the incommensurate boundary, and allows combining measurements from many micrographs into a single plot. Figure 2a shows plot of an Aubry hull function from the image in Figure 1a, and Figure 2b illustrates simulations of the same function for two rational approximants of the incommensurate structure. 
This talk will outline a procedure for the quantitative measurement of atomic relaxations at such incommensurate grain boundaries using high resolution electron microscopy, and illustrate the structure of the $90^{\circ}<110>$ tilt grain boundary in relation to its hypofriction property $[4,5]$.

\section{References}

[1] K. Westmacott et al., Philosophical Magazine A 81 (2001) 1547.

[2] F. Lancon, Europhysics Letters 57 (2002) 74.

[3] T. Kawaguchi and H. Matsukawa. Physical Review B 56 (1997) 13932

[4] F. Lancon et al., Nano Letters 10 (2010) 695

[5] This work is supported by the Director, Office of Science, Office of Basic Energy Sciences, of the U.S. Department of Energy under Contract No. DE-ACO3-76SFOOO98.

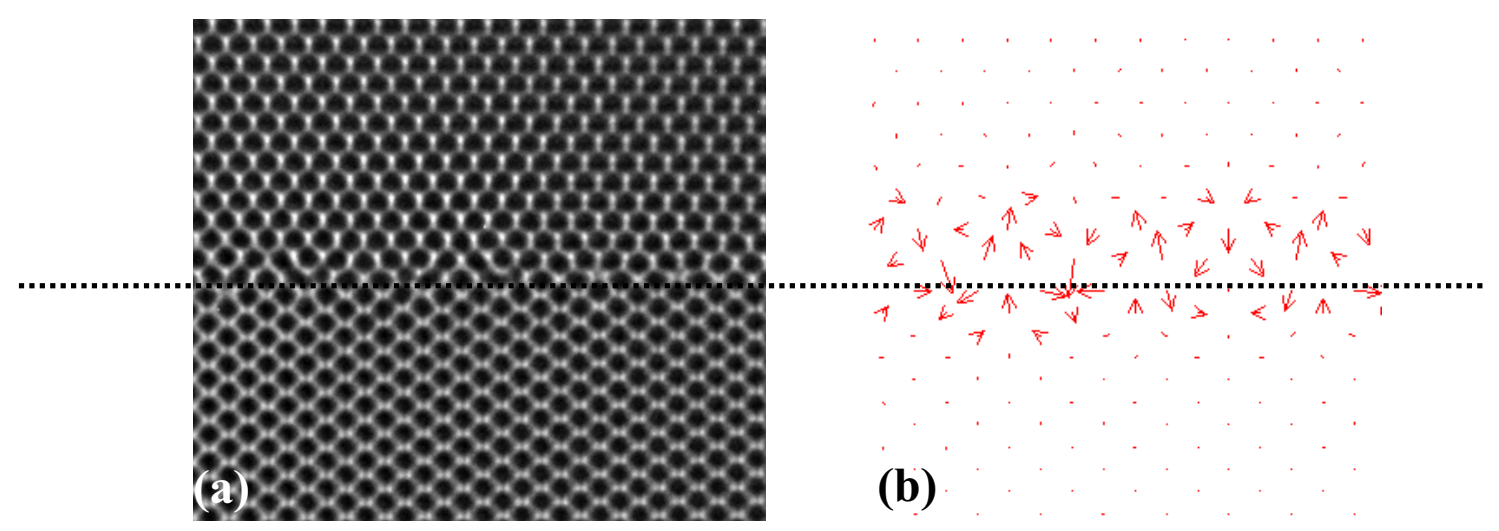

FIG. 1. (a) HREM image of a $90^{\circ}<110>$ tilt GB in Au. The periodicity of the lower grain along the grain boundary (horizontal line) is related by a factor of $\sqrt{ } 2$ to the periodicity of the upper grain. (b) magnified view of the displacement vectors representing the local shift of atomic columns with respect to their position in an unrelaxed reference lattice.
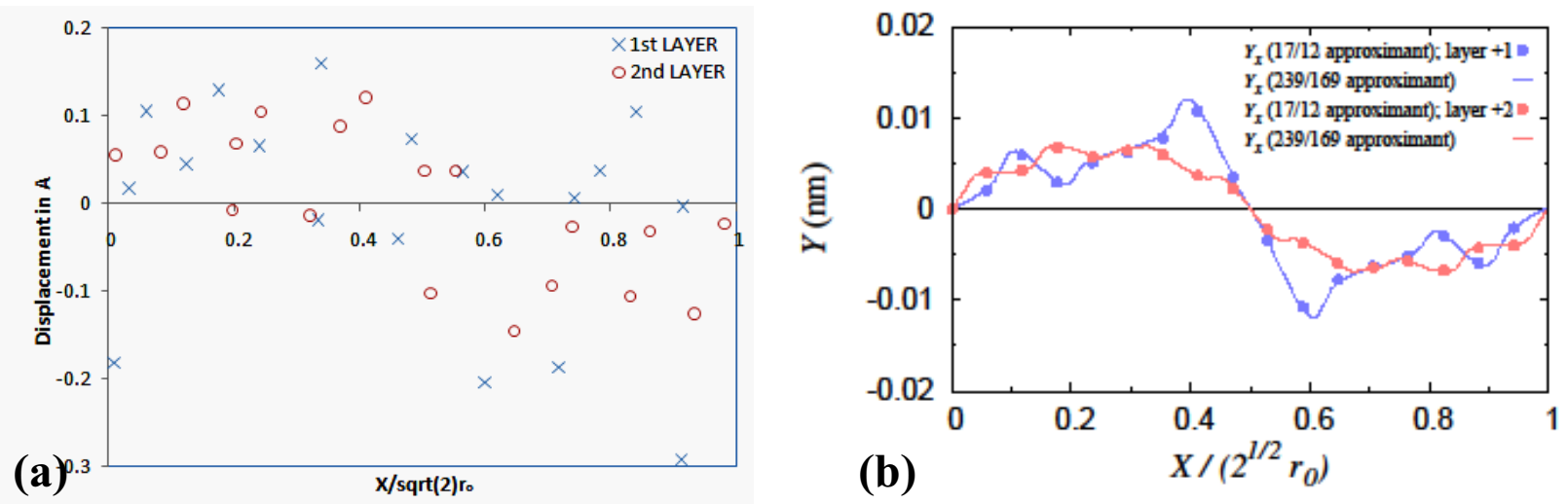

FIG. 2. Aubry hull function for the grain with periodicity $r_{o}$ (upper grain in Fig 1), (a) calculated from high resolution image shown in Fig 1. The data set represented by crosses corresponds to the atomic plane closest to the GB and the data set marked by open circles corresponds to second nearest atomic plane parallel to the GB. (b) illustrates simulations of the same function for two rational approximants of the incommensurate structure 